\title{
In Vitro Assessment of Pharmacokinetic Drug-Drug Interactions of Direct Oral Anticoagulants: Type 5-Phosphodiesterase Inhibitors Are Inhibitors of Rivaroxaban and Apixaban Efflux by P-Glycoprotein
}

\author{
Victor Margelidon-Cozzolino, Sophie Hodin, Elodie Jacqueroux, Olivier Delézay, \\ Laurent Bertoletti, and Xavier Delavenne
}

INSERM UMR 1059, Equipe Dysfonctions Vasculaires et de l' Hémostase, Faculté de Médecine de St-Etienne, Université Jean Monnet, Saint-Etienne, France (V.M.-C., S.H., E.J., O.D., X.D.); and Service de Médecine Vasculaire et Thérapeutique, CHU de St-Etienne, Saint-Etienne, France (L.B.)

Received October 30, 2017; accepted March 21, 2018

\begin{abstract}
Because of their lower bleeding risk and simplicity of use, direct oral anticoagulants (DOACs) could represent an interesting alternative to conventional anticoagulant treatment with vitamin $\mathrm{K}$ antagonists for patients with pulmonary arterial hypertension $(\mathrm{PAH})$. P-glycoprotein ( $\mathrm{P}$-gp) plays a key role in DOAC pharmacokinetics. Type 5-phosphodiesterase inhibitors (PDE5is), a drug class commonly used in the treatment of PAH, have been shown to strongly inhibit P-gp. This work aimed to assess potential P-gp-mediated drug-drug interactions between PDE5is and DOACs using in vitro methods. A cellular model of drug transport assay, using P-gp-overexpressing Madin-Darby canine kidney cells (transfected with the human P-gp gene), was used to determine the bidirectional permeabilities of two DOACs (rivaroxaban and apixaban) in the absence
\end{abstract}

and presence of increasing concentrations $(0.5-100 \mu \mathrm{M})$ of three PDE5is (sildenafil, tadalafil, and vardenafil). Permeabilities and efflux ratios were calculated from DOAC concentrations, were measured with liquid chromatography coupled with mass spectrometry, and were subsequently used to determine the PDE5i percentage of inhibition and half maximal inhibitory concentration $\left(\mathrm{IC}_{50}\right)$. Rivaroxaban efflux was inhibited by $99 \%$, $66 \%$, and $100 \%$ with $100 \mu \mathrm{M}$ sildenafil, tadalafil, and vardenafil, respectively. Similarly, apixaban efflux was inhibited by $97 \%$, $74 \%$, and $100 \%$, respectively. The $1 \mathrm{I}_{50}$ values of the three PDE5is were 8,28 , and $5 \mu \mathrm{M}$ for rivaroxaban and 23,15 , and $3 \mu \mathrm{M}$ for apixaban, respectively. This study showed strong in vitro inhibition of DOAC efflux by PDE5is. In vivo studies are required to determine the clinical relevance of these interactions.

\section{Introduction}

Anticoagulant therapy remains a challenging issue in the field of pulmonary hypertension treatment. Pulmonary arterial hypertension (PAH) is a vascular pulmonary disease characterized by elevated mean pulmonary arterial pressure that leads to right heart failure and death (Galiè et al., 2016). Oral anticoagulants, particularly vitamin $\mathrm{K}$ antagonists (VKAs), are widely used in PAH as a supportive therapy (e.g., in $60 \%$ of participants in a recent French cohort; Gabriel et al., 2016). The risk/benefit ratio of VKAs seems to be associated with a significant bleeding risk in these patients (Henkens et al., 2013).

This work was supported by La Fondation du Souffle and Le Fonds de Recherche en Santé Respiratoire.

https://doi.org/10.1124/jpet.117.245993.
In addition to anticoagulants, patients with $\mathrm{PAH}$ receive specific drugs called "targeted therapies," which include prostanoid analogs, endothelin receptor antagonists, and type 5-phosphodiesterase inhibitors (PDE5is) (Galiè et al., 2016). These targeted therapies are noticeably involved in drug-drug interactions (DDIs), especially with VKAs (Fernández and Romá, 2003; Ciracì et al., 2014; Said, 2014). Indeed, some of these drugs are P-glycoprotein (P-gp) and/or CYP3A4 substrates and can act as P-gp and CYP3A4 competitive inhibitors toward other drugs prescribed simultaneously. Interestingly, PDE5is were shown to be in vitro P-gp inhibitors when tested with chemotherapy in P-gp-overexpressing neoplastic cells (Ding et al., 2011; Shi et al., 2011). However, these studies only monitored intracellular accumulation of P-gp substrates and did not specifically study drug transport.

Direct oral anticoagulants (DOACs) are direct specific activated factor X (anti-Xa) or thrombin (Anti-IIa) inhibitors, which are increasingly prescribed for approved indications

ABBREVIATIONS: AF, atrial fibrillation; DDI, drug-drug interaction; DMSO, dimethylsulfoxide; DOAC, direct oral anticoagulation; ER, efflux ratio; FA, formic acid; FDA, U.S. Food and Drug Administration; MDCK, Madin-Darby canine kidney; m/z, mass-to-charge ratio; P-gp, P-glycoprotein; $\mathrm{PAH}$, pulmonary arterial hypertension; $\mathrm{P}_{\mathrm{app}} \mathrm{A} \rightarrow \mathrm{B}$, apical-to-basolateral permeability; $\mathrm{P}_{\mathrm{app}} \mathrm{B} \rightarrow \mathrm{A}$, basolateral-to-apical permeability; $\mathrm{PDE}$-5i, type 5-phosphodiesterase inhibitor; RER, relative efflux ratio; TEER, transepithelial electric resistance; VKA, vitamin $K$ antagonist; VTE, venous thromboembolism. 
such as venous thromboembolism (VTE) and atrial fibrillation (AF) (Gómez-Outes et al., 2015). The widespread use of DOACs is largely due to their ease of use (fixed oral doses, no need for biologic monitoring) and safer profile (fewer DDIs than VKAs, reduced major bleeding risk). Because of these advantages, DOACs could represent an interesting alternative to VKAs for anticoagulation therapy for patients with PAH (Bertoletti et al., 2013).

However, patients taking DOACs can also suffer from several DDIs, which may alter their risk/benefit ratio by increasing bleeding risk (Chang et al., 2017). DOACs are CYP3A4 (only anti-Xa) and efflux transporter P-gp substrates. More specifically, $\mathrm{P}$-gp plays a crucial role in DOAC pharmacokinetics and particularly in their intestinal absorption. As a consequence, P-gp inhibition by other drugs can deeply affect DOAC pharmacokinetics, leading to increased disposition (Bertoletti et al., 2017). Subsequently, there is a theoretical risk of DDIs between DOACs and PDE5is: PDE5is may inhibit DOAC transport by P-gp, thus increasing DOAC intestinal absorption, and may lead to increased bioavailability and bleeding risk. Among currently available DOACs, this issue seems to be a particular concern for rivaroxaban and apixaban because their dispositions are known to be highly dependent on P-gp inhibition phenomena (Bertoletti et al., 2017; Hodin et al., 2017) and trends show that their uses are becoming generalized (Loo et al., 2017).

To our knowledge, DDIs between DOAC and PAH targeted therapies have not been investigated thus far. This study aimed to assess the potential in vitro DDIs between DOACs and PDE5is by P-gp inhibition. The first objective was to determine whether three PDE5is (sildenafil, tadalafil, and vardenafil) inhibit the efflux transport by P-gp of two DOACs (rivaroxaban and apixaban). The second objective was to compare inhibitory properties of the three PDE5is tested (inhibition percentage, $\mathrm{IC}_{50}$ ) on DOAC efflux transport.

\section{Materials and Methods}

Chemical and Reagents. Apixaban, rivaroxaban, $\left[{ }^{2} \mathrm{H}_{7}^{13} \mathrm{C}\right]-$ apixaban, and $\left[{ }^{13} \mathrm{C}_{6}\right]$-rivaroxaban were purchased from Alsachim (Illkirch, France). Sildenafil, tadalafil, vardenafil, verapamil, ritonavir, formic acid (FA), and dimethylsulfoxide (DMSO) were obtained from Sigma-Aldrich (Saint-Quentin-Fallavier, France). Dulbecco's modified Eagle's medium, Hanks' balanced salt solution, Dulbecco's phosphate-buffered saline without magnesium, HEPES solution, heat-inactivated fetal bovine serum, trypsin-EDTA $(0.05 \%-0.02 \%)$, nonessential amino acids, penicillin $\mathrm{G}$, and amphotericin B streptomycin were purchased from Sigma-Aldrich.

Cell Culture. Native Madin-Darby canine kidney (MDCK) and MDCK-MDR1 cells were a generous gift from Dr. Piet Borst (Netherlands Cancer Institute, Amsterdam, Netherlands; batch DRW18 at passage 14 for MDCK-MDR1 cells, and batch DRB180 BPO/EW at passage 9 for native MDCK cells). MDCK-MDR1 cells are P-gp-overexpressing MDCK cells (cells derived from canine kidney cells transfected with the human P-gp gene).

Cells were cultured in 25-cm² flasks (Falcon, polycarbonate surface, vented cap, purchased from Dominique Dutscher, Strasbourg, France) until passage 27, containing Dulbecco's modified Eagle's medium supplemented with $10 \%$ fetal bovine serum, $1 \%$ nonessential amino acids, $100 \mathrm{U} / \mathrm{ml}$ penicillin G, $250 \mu \mathrm{g} / \mathrm{l}$ amphotericin B, and $100 \mu \mathrm{g} / \mathrm{ml}$ streptomycin, and were maintained at $37^{\circ} \mathrm{C}$ with $95 \%$ relative humidity and $5 \% \mathrm{CO}_{2}$. The culture medium was replaced every 2 days. Once cells were $80 \%$ confluent in flasks, they were seeded onto cell culture insert membranes (Falcon, high-density polyethylene terephthalate translucent membranes, $0.4 \mu \mathrm{m}$ pore size, $0.3 \mathrm{~cm}^{2}$ surface area, obtained from Dominique Dutscher) in 24-well companion plates (Dominique Dutscher) with a density of 110,000 cells per insert. The cell culture was continued in the same conditions as in the flasks, with a daily medium replacement until confluence. Sufficient cell confluence to obtain a proper epthelial barrier in each insert was assessed after 3,4 , and 5 days of culture by measuring transepithelial electric resistance (TEER). TEER was calculated according to the following formula:

$$
(R i-R b) \times S
$$

where $R i$ is the resistance in the insert, $R b$ is the resistance in the blank insert (nonseeded insert), and $S$ is the area of the cell monolayer $\left(0.3 \mathrm{~cm}^{2}\right)$. Drug transport assays were only performed on inserts with a TEER $>35 \Omega / \mathrm{cm}^{2}$.

Cell Toxicity Assay. Cell viability was assessed visually during culture in flasks and in one transparent seeded insert in each 24-well companion plate. A release lactate dehydrogenase cytotoxicity assay was performed to assess MDCK-MDR1 cell viability, with the various DOAC/PDE5i associations tested in drug transport assays, at a fixed concentration of $10 \mu \mathrm{M}$ for apixaban and rivaroxaban and at the maximal concentration of $100 \mu \mathrm{M}$ for sildenafil, tadalafil, and vardenafil (in quadruplicate per condition).

Drug Transport Assay. The MDCK-MDR1 P-gp-overexpressing cell line is one of the reference cell models for in vitro investigation of P-gp-related DDIs (http://www.fda.gov/Drugs/GuidanceComplianceRegulatoryInformation/Guidances/default.htm). The drug transport assay consisted of studying bidirectional DOAC permeability (apixaban or rivaroxaban at $10 \mu \mathrm{M}$ ) in the presence of an increasing range of PDE5is concentrations $(0,0.5,1,5,10,50$, and $100 \mu \mathrm{M})$. Transport assays were performed across a MDCK-MDR1 cell monolayer on a porous membrane separating an apical compartment from a basolateral compartment. Tested drugs were spiked in a donor compartment (either an apical or basolateral compartment) in the assay and were quantified in the opposite compartment (receiver compartment) after a 2-hour incubation of cells with drugs. Two monodirectional permeabilities, apical-to-basolateral permeability $\left(\mathrm{P}_{\mathrm{app}} \mathrm{A} \rightarrow \mathrm{B}\right)$ and basolateral-to-apical permeability $\left(\mathrm{P}_{\mathrm{app}} \mathrm{B} \rightarrow \mathrm{A}\right)$, for each condition, were computed according to the following formula (eq. 1):

$$
\mathrm{P}_{\mathrm{app}}=\frac{V_{\mathrm{r}}}{C_{0}} \times \frac{1}{S} \times \frac{C_{1}}{t}
$$

where $\mathrm{P}_{\text {app }}$ is the apparent permeability, $V_{\mathrm{r}}$ is the volume of medium in the receiver compartment, $C_{0}$ is the initial DOAC concentration in the donor compartment $(10 \mu \mathrm{M}), S$ is the area of the cell monolayer, $C_{1}$ is the DOAC final concentration in the receiver compartment, and $t$ is the incubation time ( 2 hours). Each condition was performed in triplicate.

Tested drugs were incubated in a transport buffer solution made of Hanks' balanced salt solution and HEPES (1\%). Basolateral and apical compartment volume was 0.8 and $0.7 \mathrm{ml}$, respectively. The drug transport assay started when DOACs with or without PDE5is were spiked in the donor compartment. The assay plates were incubated at $37^{\circ} \mathrm{C}$ for 2 hours. After 2 hours, the whole available volume in the receiver compartment was sampled and was rapidly analyzed in liquid chromatography coupled with mass spectrometry and then stored at $-20^{\circ} \mathrm{C}$.

Drug Solution Preparation. Stock solutions were prepared in DMSO for rivaroxaban and tadalafil and in methanol for ritonavir. Final concentrations in spiked solutions were $10 \mu \mathrm{M}$ for DOACs (rivaroxaban and apixaban) and ranged from 0.5 to $100 \mu \mathrm{M}$ for sildenafil, tadalafil, and vardenafil. Concentrations of strong P-gp inhibitors ritonavir and verapamil in final solutions were 50 and $100 \mu \mathrm{M}$, respectively. The DMSO concentration in final solutions was $1 \%$.

Liquid Chromatography-Mass Spectrometry Analysis. Quantification of DOACs was performed on an Acquity UPLC system coupled with an Xevo TQD triple quadrupole mass spectrometer 
(Waters, Saint-Quentin-en-Yvelines, France). Analysis was performed in positive ionization mode for apixaban (mass $/$ charge $[\mathrm{m} / \mathrm{z}]$ $460.23 \rightarrow 443.25)$ and rivaroxaban $(\mathrm{m} / z$ 436.11 $\rightarrow 231.22)$. The internal standards for each drug were rivaroxaban $\left[{ }^{13} \mathrm{C}_{6}\right](\mathrm{m} / z 442.11 \rightarrow 145)$ and apixaban $\left[{ }^{2} \mathrm{H}_{7}^{13} \mathrm{C}\right](\mathrm{m} / z \quad 469.3 \rightarrow 199.16)$. Fifty microliters of each sample was extracted with $300 \mu \mathrm{l}$ internal standard in methanol. For apixaban, the mobile phase comprised a mixture of (A) water containing $0.1 \% \mathrm{FA}$ and ammonium acetate $2 \mathrm{mM}$ and (B) methanol containing $0.1 \% \mathrm{FA}$ and $2 \mathrm{mM}$ ammonium acetate. For rivaroxaban, the mobile phase comprised a mixture of (A) $0.1 \%$ FA in water and (B) $0.1 \% \mathrm{FA}$ in acetonitrile. An eluate gradient was applied on a BEH C18 column $(50 \times 2.1 \mathrm{~mm} \times 2.7 \mu \mathrm{m})$ (Waters). Ratios of peak areas of drugs and their internal standards were used as $C_{0}$ and $C_{1}$ for permeability calculation.

Data Analysis. Data analyses were performed and graphics were created with R software (https://www.R-project.org/; R Foundation for Statistical Computing, Vienna, Austria). Results are expressed as the mean \pm S.D. Figures were created with R library ggplot2 (Wickham, 2009).

Efflux of DOACs by transporters across the cell monolayer was assessed by calculating the efflux ratio (ER) from the apparent permeabilities previously obtained for each condition, according to the following formula (eq. 2):

$$
\mathrm{ER}=\frac{\mathrm{P}_{\mathrm{app}} \mathrm{B} \rightarrow \mathrm{A}}{\mathrm{P}_{\mathrm{app}} \mathrm{A} \rightarrow \mathrm{B}}
$$

The tested drug is considered as an efflux transporter substrate if the value of the $\mathrm{ER}$ is superior or equal to 2 .

Inhibitory properties of PDE5is on DOAC efflux were assessed with two criteria: inhibition percentage and $\mathrm{IC}_{50}$. The inhibition percentage was calculated according to the following formula (eq. 3):

$$
\% \text { Inhibition }=\left(1-\frac{\mathrm{ER}_{\mathrm{i}}-1}{\mathrm{ER}_{\mathrm{a}}-1}\right) \times 100
$$

where $\mathrm{ER}_{\mathrm{i}}$ is the $\mathrm{ER}$ obtained with inhibitor (PDE5i) and $\mathrm{ER}_{\mathrm{a}}$ is the ER obtained without inhibitor.

The $\mathrm{IC}_{50}$ was determined from nonlinear regression modeling (function "nls" in $\mathrm{R}$ software) of the relative efflux ratio (RER) according the following equations (eqs. 4 and 5):

$$
\begin{gathered}
\mathrm{RER}=\frac{\mathrm{RE}_{\mathrm{i}}}{\mathrm{RE}_{\mathrm{a}}} \\
\frac{\mathrm{RE}_{\mathrm{i}}}{\mathrm{RE}_{\mathrm{a}}}=1-\left[\frac{I_{\max } \times I^{n}}{I^{n}+\mathrm{IC}_{50}{ }^{n}}\right]
\end{gathered}
$$

where $I$ is the PDE5i concentration, $n$ is the Hill coefficient of the sigmoid model, and $I_{\max }$ is the maximal effect of the model.

All operations were computed with $\mathrm{R}$ software. The $\left[I_{2}\right] / \mathrm{IC}_{50}$ ratio was calculated from the following formula according to U.S. Food and Drug Administration (FDA) recommendations (http://www.fda.gov/ Drugs/GuidanceComplianceRegulatoryInformation/Guidances/default. htm):

$$
\frac{\left[I_{2}\right]}{\mathrm{IC}_{50}}=\frac{D}{250} \times \frac{1}{\mathrm{IC}_{50}}
$$

where $\left[I_{2}\right]$ represents the expected PDE5i in vivo concentration at the apical side of enterocytes, $D$ is the maximal oral PDE5i dose given once (in milligrams), and $250 \mathrm{ml}$ is the mean intestinal fluid volume.

\section{Results}

Cell Viability, Cell Monolayer Integrity, and Model Assessment. The lactate dehydrogenase dosage cytotoxicity assay did not show any toxicity for any of the tested DOAC/ PDE5i associations (data not shown). The mean TEER values were $60 \pm 13$ and $50 \pm 15 \Omega / \mathrm{cm}^{2}$ before and after the assays, respectively.

Basolateral-to-apical permeabilities and ERs of rivaroxaban and apixaban decreased when associated with strong known P-gp inhibitors verapamil $(100 \mu \mathrm{M}$; ER inhibition ranged from $76 \%$ to $86 \%$ and from $78 \%$ to $88 \%$ for rivaroxaban and apixaban, respectively) and ritonavir (50 $\mu \mathrm{M}$; ER inhibition from $65 \%$ to $85 \%$ and from $66 \%$ to $92 \%$ for rivaroxaban and apixaban, respectively) (Tables 1 and 2). These results confirmed functionality of MDCK-MDR1 cells to assess inhibition of rivaroxaban and apixaban efflux by P-gp.

Efflux Ratio. Bidirectional transport assays were performed for two DOACs (rivaroxaban and apixaban) at a fixed concentration of $10 \mu \mathrm{M}$, incubated both without inhibitor and with increasing concentrations $(0.5-100 \mu \mathrm{M})$ of three PDE5is (sildenafil, tadalafil, and vardenafil). Bidirectional permeabilities and ER values of rivaroxaban and apixaban are summarized in Table 1 (rivaroxaban) and Table 2 (apixaban).

DOAC permeabilities were higher in the basolateral-toapical direction than in the apical-to-basolateral direction. Apical-to-basal permeabilities ranged from 2.7 to $3.4 \times 10^{-6}$ $\mathrm{cm} / \mathrm{s}$ for rivaroxaban and from 0.8 to $1.5 \times 10^{-6} \mathrm{~cm} / \mathrm{s}$ for apixaban. Basolateral-to-apical permeabilities ranged from 39 to $56 \times 10^{-6} \mathrm{~cm} / \mathrm{s}$ and from 26 to $41 \times 10^{-6} \mathrm{~cm} / \mathrm{s}$, respectively. DOAC ERs without inhibitor ranged from 12 to 17 for rivaroxaban and from 25 to 45 for apixaban, showing the existence of an efflux transport for these two drugs.

A dose-dependent reduction of rivaroxaban ERs was observed in the presence of increasing concentrations of PDE5is (ERs of $1.2,5.5$, and 0.8 with $100 \mu \mathrm{M}$ sildenafil, tadalafil, and vardenafil, respectively). A similar reduction was also observed for apixaban ERs $(2.5,8.0$, and 0.9 with $100 \mu \mathrm{M}$ for each of the three PDE5is, respectively). These ER reductions were due to concomitant basolateral-to-apical permeability reductions for rivaroxaban and apixaban and an apicalto-basolateral permeability increase (apical-to-basolateral permeabilities ranging from 4.9 to $14 \times 10^{-6} \mathrm{~cm} / \mathrm{s}$ and from 3.6 to $5.6 \times 10^{-6} \mathrm{~cm} / \mathrm{s}$ at $100 \mu \mathrm{M}$ PDE5i for rivaroxaban and apixaban, respectively, with basolateral-to-apical permeabilities ranging from 11 to $27 \times 10^{-6} \mathrm{~cm} / \mathrm{s}$ and from 5.0 to $28 \times 10^{-6} \mathrm{~cm} / \mathrm{s}$, respectively). ERs started to decrease with globally higher concentrations with tadalafil than with sildenafil and vardenafil (from $50 \mu \mathrm{M}$ for tadalafil, and between 1 and $10 \mu \mathrm{M}$ for both sildenafil and vardenafil). ER reductions obtained with the three tested PDE5is were similar to those obtained with strong known P-gp inhibitors verapamil and ritonavir (Table 1). ERs were stable between 2.5 and 6.8 with verapamil and between 2.8 and 10 with ritonavir.

Maximal Inhibition Assessment. The evolution of DOAC efflux inhibition with increasing PDE5i concentrations is shown in Fig. 1. Maximal inhibition ability was different between the three PDE5is at their maximal tested concentration $(100 \mu \mathrm{M})$ : sildenafil and vardenafil almost fully inhibited rivaroxaban and apixaban efflux (98\% and $100 \%$ vs. $97 \%$ and $100 \%$, respectively), whereas efflux inhibition capped at about $75 \%$ with tadalafil (66\% and $74 \%$, respectively).

$\mathrm{IC}_{50}$ and $\left[\boldsymbol{I}_{2}\right] / \mathrm{IC}_{50}$ Ratios. Modeling of RERs for the six DOACs/PDE5i combinations was performed to determine $\mathrm{IC}_{50}$ values (Fig. 2). Combinations with vardenafil showed the lowest $\mathrm{IC}_{50}$ compared with other combinations (5 and $3 \mu \mathrm{M}$ with rivaroxaban and apixaban, respectively; two to five times smaller than $\mathrm{IC}_{50}$ values found with sildenafil and tadalafil). 
TABLE 1

Rivaroxaban permeabilities and ERs for the three rivaroxaban/PDE5i combinations Data are presented as the mean \pm S.D.

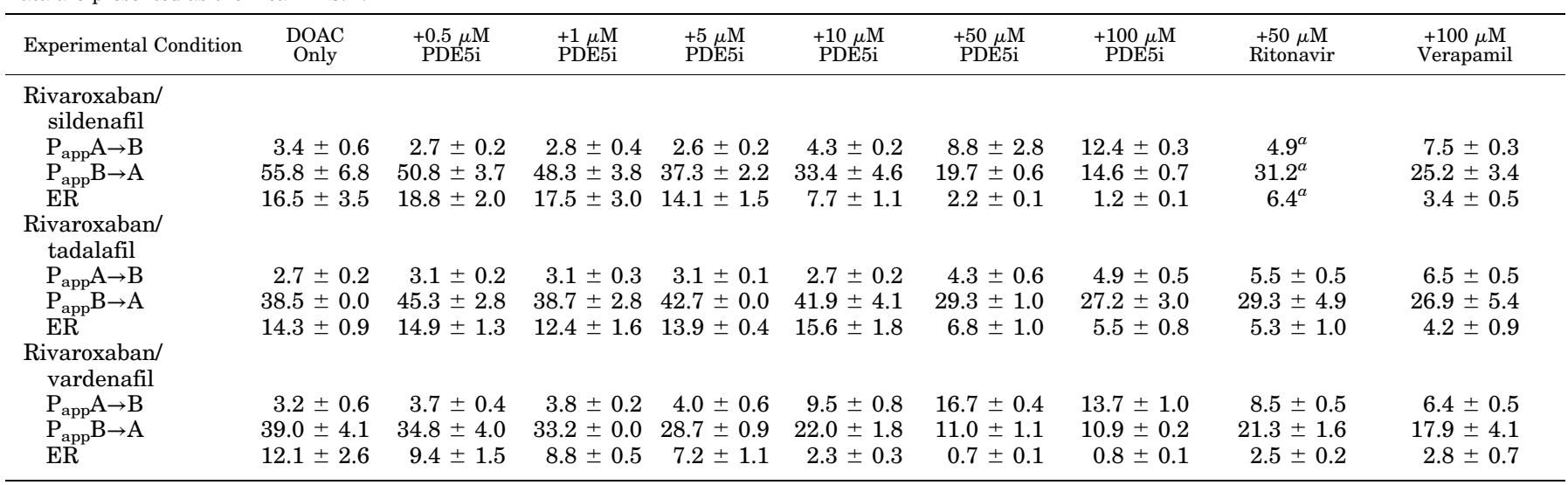

For each combination, bidirectional permeabilities $\left(\mathrm{P}_{\mathrm{app}} \mathrm{A} \rightarrow \mathrm{B}\right.$ and $\mathrm{P}_{\mathrm{app}} \mathrm{B} \rightarrow \mathrm{A}$, expressed in $10^{-6} \mathrm{~cm} / \mathrm{s}, n=3$ for both) and ERs (expressed in absolute value) of rivaroxaban are

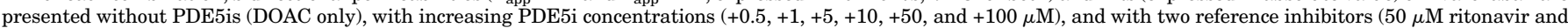
$100 \mu \mathrm{M}$ verapamil).

${ }^{a}$ No S.D. was available because this condition was performed for $n=1$ (cells on both of the other membranes were not usable according to TEER before drug transport assay).

$\mathrm{IC}_{50}$ was lower with sildenafil $(8 \mu \mathrm{M})$ than with tadalafil $(28 \mu \mathrm{M})$ for rivaroxaban efflux and conversely for apixaban efflux ( IC $_{50} 23 \mu \mathrm{M}$ vs. $19 \mu \mathrm{M}$ ).

Prediction of the clinical relevance of DDI risk can be assessed from in vitro-determined data. According to FDA guidance, we calculated $\left[I_{2}\right] / \mathrm{IC}_{50}$ ratios, which are shown in Fig. 2 beside the $\mathrm{IC}_{50}$ values. $\left[I_{2}\right]$ represents an approximation of the PDE5i expected concentrations in the intestinal tract (obtained by the ratio of PDE5i mass contained in one drug intake to the theoretical intestinal fluid volume for a standard adult; i.e., $250 \mathrm{ml}$ ). As a consequence, the $\left[I_{2}\right] / \mathrm{IC}_{50}$ ratio is a way to compare expected in vivo concentrations of PDE5is in intestinal fluid (where a part of P-gp-mediated DDIs with DOACs is supposed to occur) to a reference concentration $\left(\mathrm{IC}_{50}\right)$ around which PDE5is start to show substantial effects in in vitro models of drug transport. In other words, this ratio compares in vivo concentrations to those known to produce a relevant in vitro effect. This ratio was superior to 10 for the following combinations: rivaroxaban/sildenafil, rivaroxaban/ vardenafil, apixaban/tadalafil, and apixaban/vardenafil. This means that in vivo DDI risk might only be reasonably ruled out for both of the remaining tested combinations: rivaroxaban/tadalafil and apixaban/sildenafil.

\section{Discussion}

Because of their simplicity of use and possibly lower bleeding risk, DOACs could replace VKAs in the treatment of patients with PAH (Bertoletti et al., 2013, 2017). Although DOAcs are not currently specifically approved for anticoagulation in $\mathrm{PAH}$, some patients with $\mathrm{PAH}$ may already receive DOACs in other approved labels (AF, VTE) (Gabriel et al., 2016). Patients with PAH also receive specific therapies, some of which are already established DDI providers (mainly endothelin receptor antagonist bosentan, a CYP3A4 inducer). DOACs are P-gp substrates, and it has been previously reported that some of PDE5is, a major drug class in PAH treatment, could act as P-gp inhibitors toward

TABLE 2

Apixaban permeabilities and ERs for the three apixaban/PDE5i combinations

Data are presented as the mean \pm S.D.

\begin{tabular}{|c|c|c|c|c|c|c|c|c|c|}
\hline $\begin{array}{c}\text { Experimental } \\
\text { Condition }\end{array}$ & DOAC Only & $\begin{array}{c}+0.5 \mu \mathrm{M} \\
\text { PDE5i }\end{array}$ & $\begin{array}{l}+1 \mu \mathrm{M} \\
\text { PDE5i }\end{array}$ & $\begin{array}{l}+5 \mu \mathrm{M} \\
\text { PDE5i }\end{array}$ & $\begin{array}{c}+10 \mu \mathrm{M} \\
\text { PDE5i }\end{array}$ & $\begin{array}{c}+50 \mu \mathrm{M} \\
\text { PDE5i }\end{array}$ & $\begin{array}{c}+100 \mu \mathrm{M} \\
\text { PDE5i }\end{array}$ & $\begin{array}{c}+50 \mu \mathrm{M} \\
\text { Ritonavir }\end{array}$ & $\begin{array}{c}+100 \mu \mathrm{M} \\
\text { Verapamil }\end{array}$ \\
\hline \multicolumn{10}{|l|}{$\begin{array}{l}\text { Apixaban/ } \\
\text { sildenafil }\end{array}$} \\
\hline $\mathrm{P}_{\mathrm{app}} \mathrm{A} \rightarrow \mathrm{B}$ & $0.8 \pm 0.0$ & $1.1 \pm 0.2$ & $1.0 \pm 0.1$ & $1.3 \pm 0.1$ & $1.6 \pm 0.1$ & $2.7 \pm 0.2$ & $3.7 \pm 0.3$ & $2.7 \pm 0.2$ & $2.6 \pm 0.2$ \\
\hline $\mathrm{P}_{\mathrm{app}} \mathrm{B} \rightarrow \mathrm{A}$ & $36.5 \pm 3.5$ & $37.6 \pm 1.5$ & $28.7 \pm 1.1$ & $30.2 \pm 2.6$ & $28.0 \pm 2.9$ & $17.0 \pm 1.2$ & $9.4 \pm 1.9$ & $14.9 \pm 2.4$ & $16.6 \pm 1.0$ \\
\hline $\mathrm{ER}$ & $45.1 \pm 4.6$ & $35.6 \pm 5.6$ & $29.1 \pm 3.5$ & $23.4 \pm 2.5$ & $17.7 \pm 2.1$ & $6.3 \pm 0.6$ & $2.5 \pm 0.6$ & $5.4 \pm 1.0$ & $6.5 \pm 0.7$ \\
\hline \multicolumn{10}{|c|}{ Apixaban/tadalafil } \\
\hline $\mathrm{P}_{\mathrm{app}} \mathrm{A} \rightarrow \mathrm{B}$ & $1.5 \pm 0.2$ & $1.5 \pm 0.2$ & $1.6 \pm 0.2$ & $1.8 \pm 0.7$ & $2.5 \pm 1.0$ & $2.6 \pm 0.7$ & $3.6 \pm 0.2$ & $2.2 \pm 0.2$ & $3.6 \pm 0.7$ \\
\hline $\mathrm{P}_{\mathrm{app}} \mathrm{B} \rightarrow \mathrm{A}$ & $41.2 \pm 5.6$ & $42.1 \pm 4.9$ & $43.1 \pm 7.8$ & $35.1 \pm 2.7$ & $46.6 \pm 6.9$ & $34.4 \pm 2.0$ & $28.3 \pm 1.7$ & $22.4 \pm 2.2$ & $24.7 \pm 2.6$ \\
\hline ER & $27.8 \pm 5.9$ & $27.7 \pm 5.3$ & $27.2 \pm 5.7$ & $19.8 \pm 8.1$ & $19.1 \pm 2.9$ & $13.2 \pm 3.5$ & $8.0 \pm 0.7$ & $10.1 \pm 1.5$ & $6.8 \pm 1.5$ \\
\hline \multicolumn{10}{|l|}{$\begin{array}{l}\text { Apixaban/ } \\
\text { vardenafil }\end{array}$} \\
\hline $\mathrm{P}_{\text {app }} \mathrm{A} \rightarrow \mathrm{B}$ & $1.0 \pm 0.1$ & $1.0 \pm 0.1$ & $1.2 \pm 0.2$ & $1.9 \pm 0.1$ & $3.1 \pm 0.4$ & $4.5 \pm 0.5$ & $5.6 \pm 0.3$ & $3.6 \pm 0.7$ & $1.9 \pm 0.3$ \\
\hline $\mathrm{P}_{\mathrm{app}} \mathrm{B} \rightarrow \mathrm{A}$ & $25.8 \pm 2.8$ & $23.5 \pm 4.0$ & $22.7 \pm 2.0$ & $15.1 \pm 0.9$ & $11.3 \pm 1.2$ & $5.1 \pm 0.2$ & $5.0 \pm 0.3$ & $10.3 \pm 0.2$ & $9.4 \pm 0.9$ \\
\hline $\mathrm{ER}$ & $24.9 \pm 4.5$ & $24.4 \pm 4.4$ & $18.7 \pm 3.0$ & $8.0 \pm 0.7$ & $3.7 \pm 0.6$ & $1.1 \pm 0.1$ & $0.9 \pm 0.1$ & $2.9 \pm 0.6$ & $4.9 \pm 0.4$ \\
\hline
\end{tabular}

For each combination, bidirectional permeabilities $\left(\mathrm{P}_{\mathrm{app}} \mathrm{A} \rightarrow \mathrm{B}\right.$ and $\mathrm{P}_{\mathrm{app}} \mathrm{B} \rightarrow \mathrm{A}$, expressed in $10^{-6} \mathrm{~cm} / \mathrm{s}, n=3$ for both) and ERs (expressed in absolute value) of apixaban are

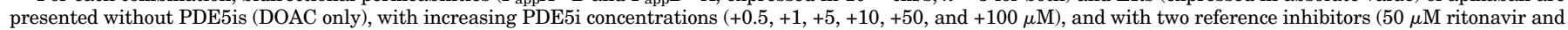
$100 \mu \mathrm{M}$ verapamil). 


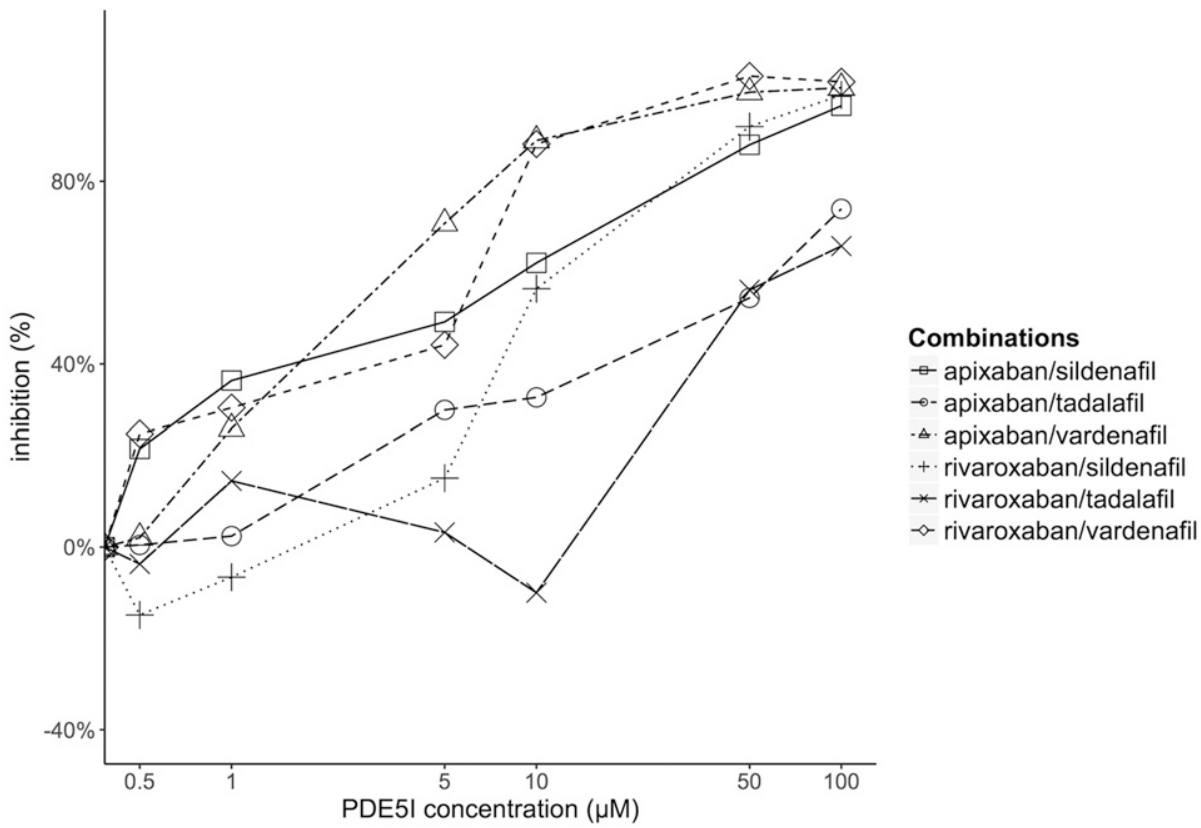

Fig. 1. Inhibitory effect of sildenafil, tadalafil, and vardenafil (expressed as the inhibition percentage) on rivaroxaban and apixaban efflux. Each DOAC/PDE5i combination is represented according to the key in the figure.

intracellular chemotherapy drugs (Ding et al., 2011; Shi et al., 2011). As a consequence, DOAC safety, particularly in terms of bleeding risk, could be compromised in this context by potential P-gp-mediated pharmacokinetic DDIs (Chang et al., 2017). To our knowledge, this work is the first to provide data on DOAC-PDE5i DDIs. This study aimed to investigate these potential interactions using in vitro methods.

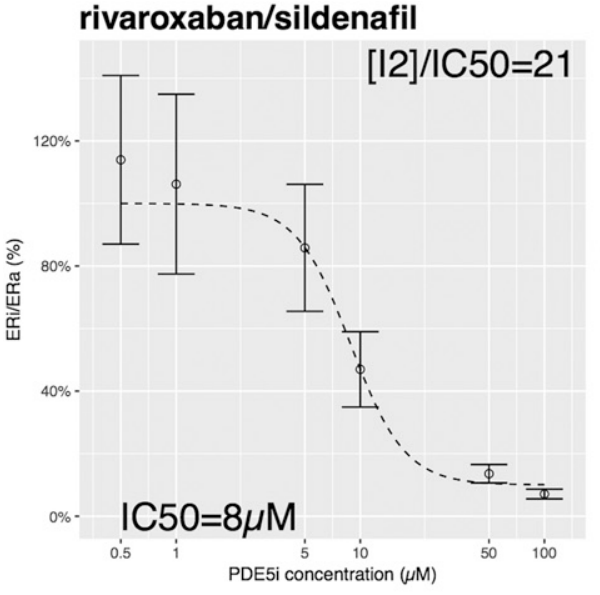

apixaban/sildenafil

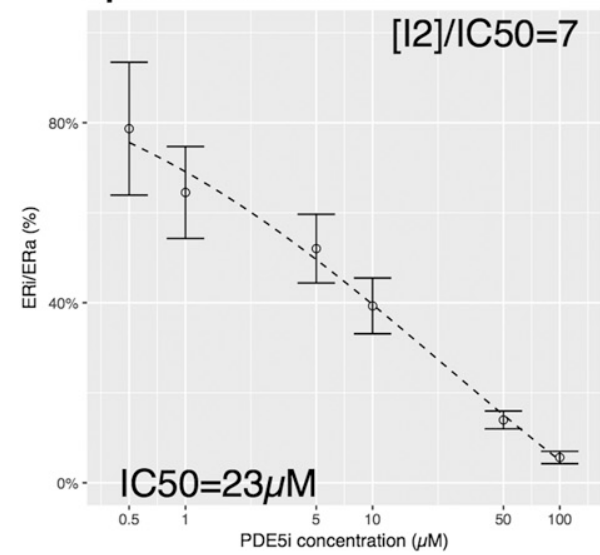

rivaroxaban/tadalafil

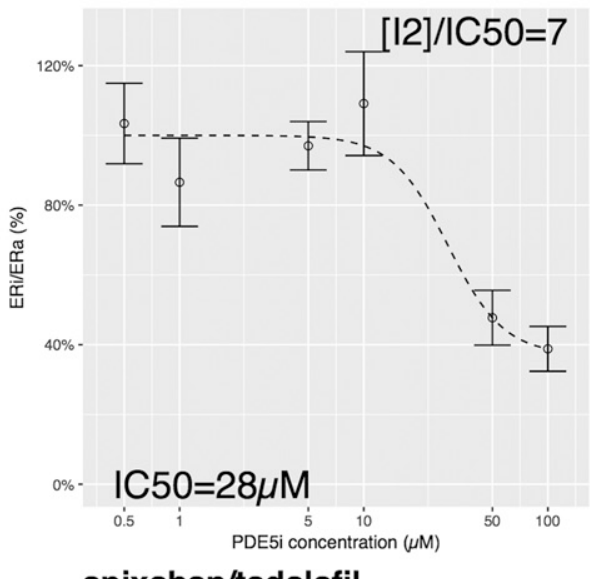

apixaban/tadalafil

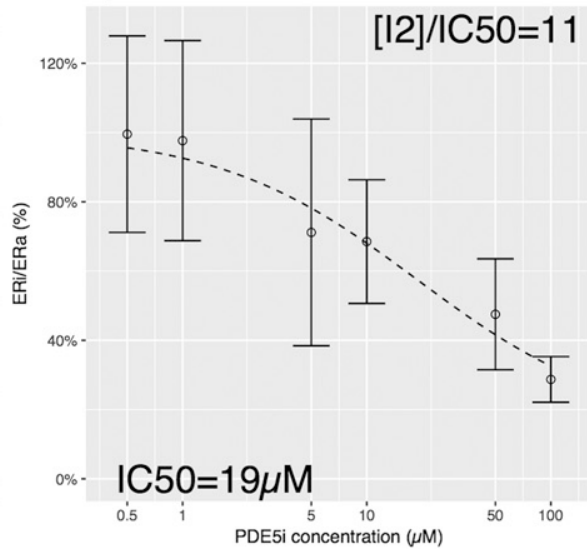

rivaroxaban/vardenafil

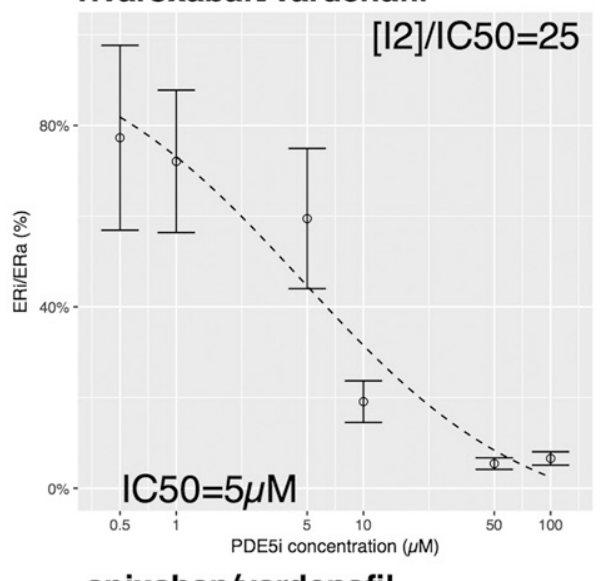

apixaban/vardenafil

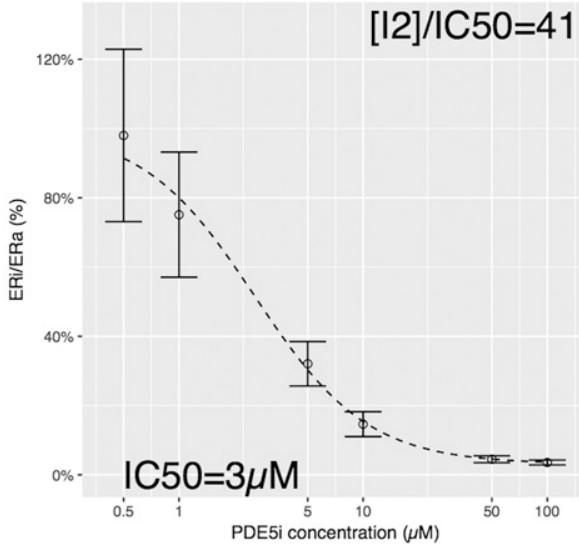

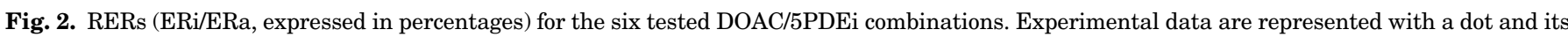

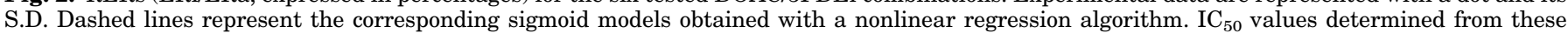
models and respective $\left[I_{2}\right] / \mathrm{IC}_{50}$ ratios are shown for each model. 
Our main results show that the three PDE5is (sildenafil, tadalafil, and vardenafil) strongly inhibit rivaroxaban and apixaban efflux across MDCK-MDR1 cells by P-gp. We found different DOAC efflux inhibition profiles for sildenafil, tadalafil, and vardenafil. At the maximal tested concentration for each PDE5i $(100 \mu \mathrm{M})$, maximal inhibition was higher with vardenafil and sildenafil (90\%-100\%) than with tadalafil (75\%) for both rivaroxaban and apixaban. In terms of DOAC efflux inhibition power, we found that vardenafil was the most powerful PDE5i $\left(\mathrm{IC}_{50}\right.$ from two to five times smaller than those found with sildenafil and tadalafil). Four DOAC-PDE5i combinations showed $\left[I_{2}\right] / \mathrm{IC}_{50}$ ratios suggesting potential clinically relevant DDIs (Fig. 2).

Among currently commercialized DOACs, we chose to test two (rivaroxaban and apixaban). These two drugs were particularly interesting to evaluate, first because they are the most sensitive DOACs to P-gp-mediated DDIs (Hodin et al., 2017) and second because they are currently the most prescribed DOACs due to their simpler use (no need for initial treatment with a parenteral anticoagulant) (Gómez-Outes et al., 2015; Loo et al., 2017). As for PDE5is, only sildenafil and tadalafil are formally approved and prescribed in the indication of PAH. Although vardenafil has not been approved for anticoagulation in $\mathrm{PAH}$, we included it in our study because it has already been evaluated in PAH and showed favorable outcomes (Galiè et al., 2016) and because previous in vitro studies suggested that this drug strongly inhibits P-gp (Ding et al., 2011).

Functionality of the cellular drug transport model we used is supported by findings from previous studies. The ER values we obtained for rivaroxaban (from 12 to 16) and apixaban (from 25 to 45) without inhibitor are consistent with those found across P-gp-overexpressing cell lines by Gnoth et al. (2011) (from 10 to 15 for rivaroxaban) and by Zhang et al. (2013) (from 23 to 38 for apixaban).

Inhibition of DOAC P-gp efflux by sildenafil, tadalafil, and vardenafil is consistent with findings from Shi et al. (2011) and Ding et al. (2011), who showed a similar effect of PDE5is on intracellular cytotoxic chemotherapy drug efflux. The plausibility of this inhibition phenomenon is also supported by the dose-response effect we found. Involvement of P-gp inhibition in the reduction of DOAC efflux is supported both by P-gp overexpression (compared with other transporters) in the cell line we used and by a similar reduction of ERs in the presence of specific P-gp strong inhibitors (verapamil and ritonavir). The differences reported between sildenafil, tadalafil, and vardenafil in terms of maximal inhibition of efflux are consistent with chemical structure differences: sildenafil and vardenafil structures differ from each other very slightly and we showed that both of these drugs almost fully inhibit rivaroxaban and apixaban efflux. Tadalafil, whose chemical structure is less similar to both of the other PDE5is, showed lower maximal inhibition of rivaroxaban and apixaban efflux (75\%). This suggests a less important affinity of tadalafil than sildenafil and vardenafil for P-gp. Previous in vitro data (Ding et al., 2011; Shi et al., 2011) similarly showed more potent inhibition with vardenafil. Nevertheless, the lesser maximal inhibition of rivaroxaban and apixaban efflux we found with tadalafil could be due to the potential incomplete range of tadalafil concentrations we studied, as a clear plateau of inhibitory effect was not reached at the maximal concentration we tested $(100 \mu \mathrm{M})$. This may have also affected the quality of the models we generated and therefore the $\mathrm{IC}_{50}$ value for tadalafil derived from these models.

The in vivo relevance of our in vitro findings can be challenged. First, we used a transfected animal cell line (MDCK-MDR1) to assess drug transport DDIs. These cells overexpress P-gp but also express animal native transporters, which can interact with our results. Theoretically, DOAC efflux inhibition found with MDCK-MDR1 cells could be partially linked to the inhibition of animal transporters. However, this seems to be a minor phenomenon, as we performed drug transport assays across native MDCK cells, which showed low involvement of animal transporters in apixaban and rivaroxaban efflux (around $12 \%$ of the $\mathrm{P}_{\text {app }} \mathrm{B} \rightarrow \mathrm{A}$ values found in MDCK-MDR1 cells) and no significant effect of PDE5is on this efflux (around 11\% of the $\mathrm{P}_{\mathrm{app}} \mathrm{B} \rightarrow \mathrm{A}$ values found in MDCK-MDR1 cells at a PDE5i concentration of $100 \mu \mathrm{M})$. One limitation to the extrapolation of our results is the poor expression of non-P-gp drug transporters by MDCKMDR1 cells, which can also be involved in DOAC efflux (e.g., Breast Cancer Resistance Protein) (Gong et al., 2013; Hodin et al., 2017). Indeed, our cell model of drug transport mainly focuses on the effect on P-gp-mediated drug transport, whereas in vivo drug transport is more complex, integrating the role of other transporters in drug pharmacokinetics. As a consequence, the in vitro observed effect of PDE5is on DOAC transport might not be significant at the in vivo scale.

Anti-Xa DOACs are also known as CYP3A4 substrates (Bertoletti et al., 2017). CYP3A4 and P-gp often have common inhibitors. What is more, CYP3A4 and P-gp are both produced and colocated in enterocytes, acting with the same purpose of preventing xenobiotics from being absorbed (Watkins, 1997; Katoh et al., 2001). PDE5is are CYP3A4 substrates. Although they are not known as clinically significant CYP3A4 inhibitors (Webb et al., 1999; Muirhead et al., 2002; Ring et al., 2005; Mora-Peris et al., 2015; Sidharta et al., 2015) toward a few specifically evaluated drugs (most of them are known as strong CYP3A4 inhibitors, such as protease inhibitors or macrolides), they have not yet been evaluated with DOACs and potential competitive inhibition between these drugs is possible. Additional data on potential CYP3A4-mediated DDIs between DOACs and PDE5is are needed to make an integrated prediction of the in vivo effect of PDE5is on DOAC pharmacokinetics.

In vitro data obtained on cell models of drug transport can provide reliable insight into the clinical relevance of DDIs. According to FDA recommendations (http://www.fda.gov/ Drugs/GuidanceComplianceRegulatoryInformation/Guidances/ default.htm), the $\left[I_{2}\right] / \mathrm{CC}_{50}$ ratio is a validated negative predictor to screen for potential clinically relevant DDIs for drugs taken orally. As mentioned in the Results, this ratio compares expected in vivo concentrations in the intestinal fluid to in vitro concentrations that have shown a substantial inhibitory effect on P-gp drug efflux. A ratio inferior to 10 indicates low probability of in vivo DDIs (not requiring further explorations), whereas values above this threshold encourage further clinical pharmacokinetic investigation (Fenner et al., 2009). In our study, only two combinations showed $\left[I_{2}\right] / / \mathrm{IC}_{50}$ ratios inferior to 10 , suggesting that the four remaining combinations may produce clinically relevant DDIs (Fig. 2). However, this is a simplified method and other approaches such as integrative physiologically based pharmacokinetic models can be used to make such predictions. This is 
particularly true since phenomena that occur in vivo, such as protein binding, are not considered in this model (in vivo, PDE5is have shown strong plasma protein binding, superior to $90 \%$ ). Moreover, $\left[I_{2}\right]$ is a very approximate estimation of drug solubility in intestinal fluid. Finally, other methods of drug delivery (i.e., other than oral administration, like intravenous administration) may allow to bypass intestinal absoprtion step, and thus to avoid P-gp-mediated DDIs at the apical side of enterocytes.

In summary, PDE5is are in vitro inhibitors of P-gp-mediated efflux of two DOACs (rivaroxaban and apixaban). Differences seem to exist between sildenafil, tadalafil, and vardenafil in terms of maximal inhibition and inhibition potency. The PDE5i that was least likely to interact (with the highest $\left[I_{2}\right] / \mathrm{IC}_{50}$ ratio) depended on which DOAC was tested. Pharmacokinetic in vivo studies are needed to assess the clinical relevance of these in vitro DDIs. This would be useful to select the PDE5i/DOAC combinations with the least risk of interaction. This is a particularly relevant issue as DOACs are increasingly prescribed for their safe profile and poorly constrained use in VTE and AF treatment, two conditions that are present concomitantly in nearly half of patients with PAH (Gabriel et al., 2016). At the least, particular attention should be paid to assessing bleeding risk in patients with $\mathrm{PAH}$ receiving DOACs and PDE5is simultaneously. Further data on DDI risk with other PAH targeted therapies will also be required.

\section{Acknowledgments}

Native MDCK and MDCK-MDR1 cells were a generous gift from Dr. Piet Borst.

\section{Authorship Contributions}

Participated in research design: Margelidon-Cozzolino, Hodin, Jacqueroux, Bertoletti, Delavenne.

Conducted experiments: Margelidon-Cozzolino, Hodin.

Performed data analysis: Margelidon-Cozzolino, Hodin, Delavenne.

Wrote or contributed to the writing of the manuscript: MargelidonCozzolino, Hodin, Jacqueroux, Delézay, Bertoletti, Delavenne.

\section{References}

Bertoletti L, Delavenne X, and Montani D (2013) Antithrombotics in pulmonary hypertension: more work needed before we turn to newer agents! Eur Respir J 41: $775-777$.

Bertoletti L, Ollier E, Duvillard C, Delavenne X, Beyens MN, De Magalhaes E, Bellet F, Basset T, Mismetti P, and Laporte S (2017) Direct oral anticoagulants: current indications and unmet needs in the treatment of venous thromboembolism. Pharmacol Res 118:33-42.

Chang S-H, Chou IJ, Yeh YH, Chiou MJ, Wen MS, Kuo CT, See LC, and Kuo CF (2017) Association between use of non-vitamin K oral anticoagulants with and without concurrent medications and risk of major bleeding in nonvalvular atrial fibrillation. JAMA 318:1250-1259.

Ciracì R, Tirone G, and Scaglione F (2014) The impact of drug-drug interactions on pulmonary arterial hypertension therapy. Pulm Pharmacol Ther 28:1-8.

Ding PR, Tiwari AK, Ohnuma S, Lee JWKK, An X, Dai CL, Lu QS, Singh S, Yang $\mathrm{DH}$, Talele TT, et al. (2011) The phosphodiesterase-5 inhibitor vardenafil is a potent inhibitor of ABCB1/P-glycoprotein transporter. PLoS One 6:e19329.
Fenner KS, Troutman MD, Kempshall S, Cook JA, Ware JA, Smith DA, and Lee CA (2009) Drug-drug interactions mediated through P-glycoprotein: clinical relevance and in vitro-in vivo correlation using digoxin as a probe drug. Clin Pharmacol Ther 85:173-181.

Fernández MA and Romá E (2003) International normalized ratio (INR) increase in patients taking oral anticoagulant therapy (OAT) and using sildenafil (Viagra). Haematologica 88:ELT34.

Gabriel L, Delavenne X, Bedouch P, Khouatra C, Bouvaist H, Cordier JF, Mornex JF, Pison C, Cottin V, and Bertoletti L (2016) Risk of direct oral anticoagulant bioaccumulation in patients with pulmonary hypertension. Respiration 91:307-315.

Galiè N, Humbert M, Vachiery JL, Gibbs S, Lang I, Torbicki A, Simonneau G, Peacock A, Vonk Noordegraaf A, Beghetti M, et al. (2016) 2015 ESC/ERS guidelines for the diagnosis and treatment of pulmonary hypertension. Eur Heart $J$ 37:67-119.

Gnoth MJ, Buetehorn U, Muenster U, Schwarz T, and Sandmann S (2011) In vitro and in vivo P-glycoprotein transport characteristics of rivaroxaban. J Pharmacol Exp Ther 338:372-380.

Gómez-Outes A, Suárez-Gea ML, Lecumberri R, Terleira-Fernández AI, and VargasCastrillón E (2015) Direct-acting oral anticoagulants: pharmacology, indications, management, and future perspectives. Eur J Haematol 95:389-404.

Gong IY, Mansell SE, and Kim RB (2013) Absence of both MDR1 (ABCB1) and breast cancer resistance protein (ABCG2) transporters significantly alters rivaroxaban disposition and central nervous system entry. Basic Clin Pharmacol Toxicol 112: 164-170.

Henkens IR, Hazenoot T, Boonstra A, Huisman MV, and Vonk-Noordegraaf A (2013) Major bleeding with vitamin $\mathrm{K}$ antagonist anticoagulants in pulmonary hypertension. Eur Respir J 41:872-878.

Hodin S, Basset T, Jacqueroux E, Delezay O, Clotagatide A, Perek N, Mismetti P, and Delavenne X (2017) In vitro comparison of the role of P-glycoprotein and breast cancer resistance protein on direct oral anticoagulant disposition. Eur J Drug Metab Pharmacokinet 91:307-315.

Katoh M, Nakajima M, Yamazaki H, and Yokoi T (2001) Inhibitory effects of CYP3A4 substrates and their metabolites on P-glycoprotein-mediated transport. Eur $J$ Pharm Sci 12:505-513.

Loo SY, Dell'Aniello S, Huiart L, and Renoux C (2017) Trends in the prescription of novel oral anticoagulants in UK primary care. Br J Clin Pharmacol 83:2096-2106. Mora-Peris B, Else L, Goldmeier D, Mears A, Weston R, Cooke G, Khoo S, Back D, and Winston A (2015) A phase I study to assess the safety, tolerability and pharmacokinetic profile of boceprevir and sildenafil when dosed separately and together, in healthy male volunteers. J Antimicrob Chemother 70:1812-1815.

Muirhead GJ, Faulkner S, Harness JA, and Taubel J (2002) The effects of steadystate erythromycin and azithromycin on the pharmacokinetics of sildenafil in healthy volunteers. $\mathrm{Br} J$ Clin Pharmacol 53 (Suppl 1):37S-43S.

Ring BJ, Patterson BE, Mitchell MI, Vandenbranden M, Gillespie J, Bedding AW, Jewell H, Payne CD, Forgue ST, Eckstein J, et al. (2005) Effect of tadalafil on cytochrome P450 3A4-mediated clearance: studies in vitro and in vivo. Clin Pharmacol Ther 77:63-75.

Roldan T, Landzberg MJ, Deicicchi DJ, Atay JK, and Waxman AB (2016) Anticoagulation in patients with pulmonary arterial hypertension: an update on current knowledge. J Heart Lung Transplant 35:151-164.

Said K (2014) Anticoagulation in pulmonary arterial hypertension: contemporary data from COMPERA registry. Glob Cardiol Sci Pract 2014:48-52.

Shi Z, Tiwari AK, Shukla S, Robey RW, Singh S, Kim IW, Bates SE, Peng X, Abraham I, Ambudkar SV, et al. (2011) Sildenafil reverses ABCB1- and ABCG2mediated chemotherapeutic drug resistance. Cancer Res 71:3029-3041.

Sidharta PN, Krähenbühl S, and Dingemanse J (2015) Pharmacokinetic and pharmacodynamic evaluation of macitentan, a novel endothelin receptor antagonist for the treatment of pulmonary arterial hypertension. Expert Opin Drug Metab Toxicol 11:437-449.

Watkins PB (1997) The barrier function of CYP3A4 and P-glycoprotein in the small bowel. Adv Drug Deliv Rev 27:161-170.

Webb DJ, Freestone S, Allen MJ, and Muirhead GJ (1999) Sildenafil citrate and blood-pressure-lowering drugs: results of drug interaction studies with an organic nitrate and a calcium antagonist. Am J Cardiol 83 (5A):21C-28C.

Wickham H (2009) ggplot2: Elegant Graphics for Data Analysis, Springer-Verlag, New York.

Zhang D, He K, Herbst JJ, Kolb J, Shou W, Wang L, Balimane PV, Han YH, Gan J, Frost CE, et al. (2013) Characterization of efflux transporters involved in distribution and disposition of apixaban. Drug Metab Dispos 41:827-835.

Address correspondence to: Dr. Xavier Delavenne, Laboratoire PIB, Sainbiose, Campus Santé Innovation, 10 rue de la Marandière, 42270 SaintPriest-en-Jarez, France. E-mail: xavier.delavenne@chu-st-etienne.fr 